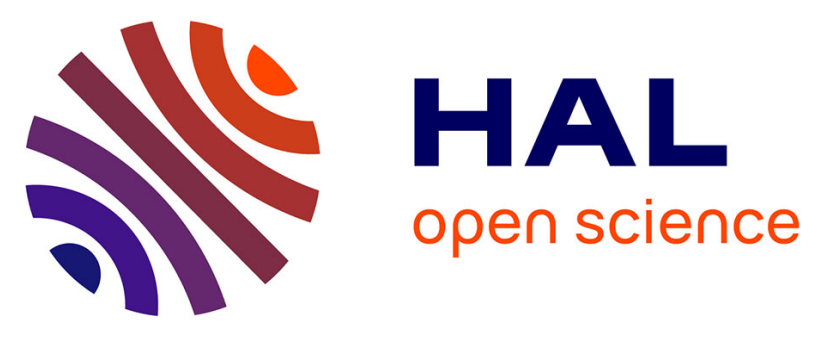

\title{
Angular Based Beamforming and Power Allocation Framework in a Multi-User Millimeter-Wave Massive MIMO System
}

Mohamed Shehata, Maryline Hélard, Matthieu Crussière, Antoine Roze, Charlotte Langlais

\section{To cite this version:}

Mohamed Shehata, Maryline Hélard, Matthieu Crussière, Antoine Roze, Charlotte Langlais. Angular Based Beamforming and Power Allocation Framework in a Multi-User Millimeter-Wave Massive MIMO System. 2018 IEEE 87th Vehicular Technology Conference: VTC2018-Spring, Jun 2018, Porto, Portugal. 10.1109/vtcspring.2018.8417520 . hal-01730747

\section{HAL Id: hal-01730747 \\ https://hal.science/hal-01730747}

Submitted on 13 Mar 2018

HAL is a multi-disciplinary open access archive for the deposit and dissemination of scientific research documents, whether they are published or not. The documents may come from teaching and research institutions in France or abroad, or from public or private research centers.
L'archive ouverte pluridisciplinaire HAL, est destinée au dépôt et à la diffusion de documents scientifiques de niveau recherche, publiés ou non, émanant des établissements d'enseignement et de recherche français ou étrangers, des laboratoires publics ou privés. 


\title{
Angular Based Beamforming and Power Allocation Framework in a Multi-User Millimeter-Wave Massive MIMO System
}

\author{
M. Shehata ${ }^{1}$, M. Hélard ${ }^{1}$, M. Crussière ${ }^{1}$, A. Rozé Re $^{1,2}$ and C. Langlais ${ }^{3}$ \\ ${ }^{1}$ Univ Rennes, INSA Rennes, CNRS, IETR - UMR 6164, F-35000 Rennes, France \\ ${ }^{2}$ IRT $b<>$ com, Rennes, France \\ ${ }^{3}$ Institut Mines-Telecom, Telecom Bretagne, CNRS, Lab-STICC - UMR 6285, F-29200 Brest, France
}

\begin{abstract}
Millimeter-Wave (mmWave) systems offer extremely large Band-Width (BW) and highly Line-of-Sight (LOS) dominant channels. Many beamforming and power allocation techniques have recently emerged to leverage such channel characteristics in order to enhance the sum capacity and the coverage for multi-user transmissions. However, most of the existing algorithms are practically limited due to full channel knowledge and high complexity requirements. In this paper we introduce a novel, low complexity, angular based beamforming and power allocation framework, that requires the knowledge of the main contributive Directions of Arrival/Departure (DoAs/DoDs) of the propagation channel only. Exploiting the fact that propagation conditions are highly driven by the geometrical structure of the channel in mmWave scenarios, our method relies on the estimation of the leakage caused by each User Equipment (UE) on all the other UEs, approximated from the DoAs contributions. We prove with the simulation results that this approach, in addition to be practically plausible, can enhance the network Spectral Efficiency (SE) alongside with maintaining acceptable data rates for the cell edge UEs.
\end{abstract}

Index Terms-Massive MIMO, mmWave, Digital beamsteering, Leakage based power allocation, LOS channel, DoA

\section{INTRODUCTION}

In the recent years, wireless communication networks started witnessing a growing demand for higher data rates and better coverage [1]. Massive MIMO [2] mmWave [3] systems have evolved as win-win key enablers for the future generation of wireless networks $(5 \mathrm{G})$ to support higher achievable data rates. Due to the wide Band-Width (BW) and high antenna gains, Massive MIMO mmWave systems are able to achieve very high per User Equipment (UE) peak data rates of tens of Gigabits per second (Gbps).

Moreover, densely deploying small cells with high data rate requirement and small per cell coverage area encourages using new solutions adapted to Line-of-Sight (LOS) channels. Accordingly, today it is well recognized that the future networks should embed a wireless component relying on the joint benefit of mmWaves, massive MIMO and small cells.

However, working at several tens of Gigahertz imposes a lot of hardware and channel implications that need to be tackled in order to unleash the high potentials of massive MIMO [4]. From a baseband signal processing point of view, efficient usage of large antenna arrays through digital beamforming techniques attracts huge interest in the perspective of improved Energy Efficiency (EE) and Spectral Efficiency (SE) using smart multi-user separation in the spatial domain. However, a central issue is to ensure the availability of the Channel State Information (CSI) of the Multi-User MIMO (MU-MIMO) channel at the transmitter upon which digital beamforming strategies are based.

Many optimization algorithms have highlighted that power allocation in MU MIMO [5], [6] and MU Massive MIMO [7] systems, is an additional processing layer which can lead to substantial improvements of the system's SE and EE. However, these algorithms focus only on optimizing the objective function, without considering the computational complexity, latency and overhead constraints. Complexity problems have partially been addressed in [8], [9] where the authors focus their effort on improving the performance of low complexity linear precoders such as the Conjugate Beamforming (CB). It is interestingly shown that such simple precoders can achieve considerable gains in $\mathrm{SE}$ and $\mathrm{EE}$ when coupled together with an interference wise power allocation. Though, the proposed iterative algorithms can't scale with Massive MIMOultra dense networks' low latency-low overhead requirements.

Contrary to the aforementioned algorithms, some other interesting perspectives appeared with introducing the Leakage Based Power Allocation (LB-PA) [10] solution. Such approach is a low complexity, closed form non iterative one. It aims at minimizing the interference each UE generates to all the other UEs which is referred to as leakage. Such an approach is interesting to pursue together with finding an answer to the channel estimation overhead minimization.

In previous work [11], [12], we introduced the Digital BeamSteering (DBS) precoding concept as a simple frequency flat - angle based beamforming technique which can be viewed as the MU extension of the Analog Beam-Steering (ABS). It is considered an interesting paradigm for the mmWave Massive MIMO systems due to its low complexity and low overhead. In the case of a propagation scenario strongly conditioned by the LOS component of the channel, as is the case in mmWave and/or in the small-cell environment, it is favorable to implement a precoder such as DBS which maximizes the emitted power only in the main direction of each user. In this paper, we investigate the potentials of DBS precoding through coupling it with an interference wise power allocation in a hierarchical framework. More precisely, we propose to couple DBS with LB-PA in order to ensure the low complexity of our scheme. Moreover, we derive closed form simplifications for the LB-PA to adapt it to the DBS low overhead. Thus, in our introduced approach 
the DoAs information is only required and the full Channel State Information at the Transmitter (CSIT) requirement is relaxed.

Therefore, contribution of the introduced framework in this paper compared to the previous Massive MIMO beamforming and power allocation algorithms in the literature can be summarized as follows:

- Angular Analysis: We present a DBS - LB-PA framework that depends only on DoA information with multiple simplifications and evaluate the performance in each case. Thus, relaxing the full CSI requirement.

- Scalability: Due to the low complexity of our solution it can scale with increasing the number of antennas and it can be implemented at sophisticated mmWave Digital Signal Processing (DSP). Also, due to the low overhead, our solution can be used for coordination between cells in multi-cell environments through relaxing the backhaul constraints.

- Coverage and Outage Analysis: In this paper we provide analysis for the coverage achieved by the DBS - LB-PA. We prove by simulation results that it achieves low outage probability together with enhancing the sum SE.

For baseline scenarios, we decouple the beamforming and power allocation problems in order to ensure a flexible framework. We consider two classical precoding techniques as a baseline which are the Zero Forcing (ZF) and the CB precoders. Then we consider DBS as our contribution. For the power allocation problem we consider the LB-PA approach. First we utilize LB-PA based on full CSI. Afterwards, we relax the full CSI requirement and derive a simpler power allocation strategy that relies only on the DoA information. Then we show by simulation results how our proposed angular frequency flat framework (DBS - LB-PA with DoA information) can achieve high data rates in highly LOS environments with low overhead and low complexity requirements.

\section{SYSTEM MODEL}

We consider a downlink MIMO system with $M$ transmit antennas at the BS and a set of $K$ UEs. Precoding techniques are applied at the transmit side in Space Division Multiple Access (SDMA) mode. Each UE is assumed to have only a single receive antenna. Let $\mathbf{s}=\left[S_{1}, S_{2}, \ldots, S_{K}\right]^{T} \in \mathbb{C}^{K \times 1}$ denote the vector of the symbols to be transmitted to the $K$ UEs, $S_{k}$ being dedicated to UE k, k $\in\{1, K\}$. $\mathbf{x}=\left[X_{1}, X_{2}, \ldots, X_{M}\right]^{T} \in \mathbb{C}^{M \times 1}$ denote the vector of the $M$ symbols emitted from the $M$ transmit antennas, which are obtained after precoding expressed by the matrix $\mathbf{W} \in \mathbb{C}^{M \times K}$ such that:

$$
\mathbf{x}=\sqrt{\eta} \mathbf{W} \mathbf{s},
$$

where $\eta=\frac{1}{\mathbb{E}\left[\left(\operatorname{Tr}\left(\mathbf{W}^{H} \mathbf{W}\right)\right]\right.}$ is the power normalization factor. The vector $\mathbf{r}=\left[R_{1}, R_{2}, \ldots, R_{K}\right]^{T} \in \mathbb{C}^{K \times 1}$ represents the symbols received by each of the $K$ UEs, where $R_{k}$ is the symbol received by UE $\mathrm{k}$. It can be expressed as:

$$
\mathbf{r}=\mathbf{G} \mathbf{x}+\mathbf{n},
$$

where $\mathbf{n}=\left[N_{1}, N_{2}, \ldots, N_{K}\right]^{T} \in \mathbb{C}^{K \times 1}$ is the vector of independent and identicaly distributed (i.i.d.) Additive White Gaussian Noise (AWGN) of zero mean and variance $\mathbb{E}\left[N_{k}^{*} N_{k}\right]=\sigma^{2}, \forall k \in[1, K]$. While $\mathbf{G} \in \mathbb{C}^{K \times M}$ is the channel matrix between the $M$ transmit antennas and the $K$ UEs.

A perfect Orthogonal Frequency Division Multiplexing (OFDM) system is assumed. Thus, the channel is represented by one different
Table I

PRECODING SUMMARY

\begin{tabular}{|c|c|c|c|}
\hline Precoder & CB & ZF & DBS \\
\hline $\mathbf{W}$ & $\mathbf{H}^{H}$ & $\mathbf{H}^{H}\left(\mathbf{H H}^{H}\right)^{-1}$ & $\mathbf{A}_{\mathbf{1}}{ }^{H}$ \\
\hline
\end{tabular}

matrix for each sub-carrier. $G_{k, m}$ represents a flat channel between the transmit antenna of index $m \in\{1, M\}$ and the UE $k$. Thus, the channel matrix $\mathbf{G}$ can be expressed as:

$$
\mathbf{G}=\mathbf{D}_{\beta}^{1 / 2} \odot \mathbf{H}
$$

$\mathbf{H} \in \mathbb{C}^{K \times M}$ is the small scale fading matrix with $\mathbb{E}\left[\left|H_{k, m}\right|^{2}\right]=1$, and $\mathbf{D}_{\beta} \in \mathbb{C}^{K \times M}$ is the large scale fading matrix with coefficients $\beta_{k, m}$. Thus, for each sub-carrier, and for each link between transmit antenna $m$ and UE $k$ of size $d_{k, m}$, we have:

$$
G_{k, m}=\sqrt{\beta}_{k, m} H_{k, m},
$$

and $\beta_{k, m}$ is calculated as follows:

$$
\beta_{k, m}=\left(\frac{\lambda}{4 \pi d_{k, m}}\right)^{2}
$$

where $\lambda$ is the wavelength. In mmWave systems, the distance between the BS and the UEs is very large compared to the distance between the transmit antennas of the BS. Henceforth, all the distances $d_{k, m}$ can be assumed equal for all the $M$ transmit antennas. Thus, the term $\beta_{k, m}$ can be replaced by $\beta_{k}$, and the term $m$ can be omitted for readability $\forall m \in[1, M]$. Therefore, the channel matrix $\mathbf{G}$ can be expressed as:

$$
\mathbf{G}=\mathbf{D}_{\beta}^{1 / 2} \mathbf{H}
$$

where $\mathbf{D}_{\beta} \in \mathbb{C}^{K \times K}$ becomes a diagonal matrix. Each diagonal element $\beta_{k}$ in $\mathbf{D}_{\beta}$ is the large scale fading between the BS and UE $k$.

\section{Angular Channel AND BEAmForming}

As aforementioned we consider three precoders in this paper which are $\mathrm{ZF}, \mathrm{CB}$ and DBS. Their respective precoding matrices are described in more details in [12] and summed up in Table I. The channel vector between UE $k$ and the $M$ antennas of the BS can be written as follows on a flat channel:

$$
\mathbf{g}_{k}=\sum_{l=1}^{L_{k}} \sqrt{\beta_{k, l}} \mathbf{a}_{k, l} e^{j \phi_{k, l}},
$$

with $\beta_{k, l}$ and $\phi_{k, l}$ being respectively the path attenuation and the phase shift of path $l$ for user $k$. Henceforth, the steering vector $\mathbf{a}_{k, l}$ can be expressed as follows:

$$
\begin{aligned}
\mathbf{a}_{k, l}=\left[1, \ldots, e^{j\left(M_{C}-1\right) \frac{2 \pi}{\lambda} d \cos \left(\varphi_{k, l}\right) \sin \left(\theta_{k, l}\right)}, \ldots,\right. & \\
& \left.e^{j \frac{2 \pi}{\lambda} d\left(\left(M_{C}-1\right) \cos \left(\varphi_{k, l}\right) \sin \left(\theta_{k, l}\right)+\left(M_{R}-1\right) \sin \left(\varphi_{k, l}\right)\right)}\right]^{T},
\end{aligned}
$$

where $\theta_{k, l}$ and $\varphi_{k, l}$ are the azimuth and elevation angles respectively. $M_{R}$ and $M_{C}$ are the number of rows and columns of the antenna array, respectively with $M_{C} \times M_{R}=M . \mathbf{A}_{l}=\left[\mathbf{a}_{1, l}, \mathbf{a}_{2, l}, \ldots, \mathbf{a}_{K, l}\right]^{T} \in$ 
$\mathbb{C}^{K \times M}$ is the steering matrix of path $l$. If we assume the channel is purely LOS, the channel matrix simplifies to:

$$
\stackrel{\operatorname{los}}{\mathbf{G}}=\sqrt{\beta_{k, 1}} e^{j \phi_{k, 1}} \mathbf{A}_{1} .
$$

\section{INTERFERENCE AND LEAKAGE ANALYSIS}

High directivity beamforming with narrow beams can achieve high beamforming gains, but are however very sensitive to channel knowledge inaccuracies at the transmitter. In techniques like ZF for instance, the nulling positions are very sensitive to channel estimation errors in high directivity cases. Henceforth, other beamforming techniques like CB and DBS can become more favorable than ZF in practical Massive MIMO regime due to the less sensitivity to estimation errors with high directivity. However, due to the fact that CB and DBS don't consider minimizing the interference between the UEs, we focus on adding an extra processing layer for suppressing it.

In this paper we utilize the LB-PA for suppressing the interference through power allocation processing for precoders that don't consider interference as an objective. In order to utilize the LB-PA we have first to define the leakage metric which will be considered the main building block of the algorithm. The symbol $R_{k}$ received by UE $k$ can be formulated as:

$$
R_{k}=\mathbf{g}_{k}^{T} \mathbf{w}_{k} S_{k}+\sum_{i=1 \neq k}^{K} \mathbf{g}_{k}^{T} \mathbf{w}_{i} S_{i}+N_{k},
$$

where UE $k$ is allocated power $\mathbb{E}\left[\left|S_{k}\right|^{2}\right]=P_{k} . \mathbf{w}_{k}$ is the $k^{\text {th }}$ column of the precoding matrix $\mathbf{W}$. If we analyze the symbol $R_{k}$ received by the user $k$, we notice that the interfering part is the sum of the $K-1$ signals destined for the other UEs and weighted by their respective precoders $\left(\mathbf{w}_{i}, \forall i \neq k \in[1, K]\right)$.

From the BS's perspective, it is relevant to know how the formation of a beam to a new UE will harm its neighbors (i.e. the leakage that will be caused). We denote the leakage created by the transmission to UE $k$ as $I_{k}$. OFDM transmission is assumed with $N_{F F T}$ subcarriers. In the general case of $M$ transmit antennas and $N_{F F T}$ subcarriers, the Leakage resulting from the transmission to UE $k$ is calculated as follows:

$$
I_{k}=\frac{P_{k}}{N_{F F T}} \sum_{n=1}^{N_{F F T}} \sum_{j \neq k=1}^{K}\left|\mathbf{g}_{j}[n]^{T} \mathbf{w}_{k}[n]\right|^{2},
$$

where $\mathbf{g}_{j}[n]$ is the vector comprising, for the subcarrier $n$, the $M$ frequency attenuating coefficients associated with the $M$ antenna-toantenna links between the transmitter and the $j^{\text {th }}$ receiver. Similarly, $\mathbf{w}_{k}[n]$ is the precoding vector constructed from the attenuation coefficients for the subcarrier $n$ of the channels between the UE $k$ and the BS.

From this expression, it is clear that the calculation of Leakage requires a complete knowledge of the channel response constructed from the frequency responses of the set of links (ie $M \times K$ links), for all the subcarriers. The number of subcarriers to be estimated depends closely on the BW and the coherence band. Let $N_{B c}$ be the number of subcarriers in the coherence band, then, the number of coefficients to be estimated to obtain the channel frequency response is $\frac{M \times K \times N_{F F T}}{N_{B c}}$.

The constraint of complete knowledge of the channel response becomes problematic in Massive MIMO mmWave systems, since

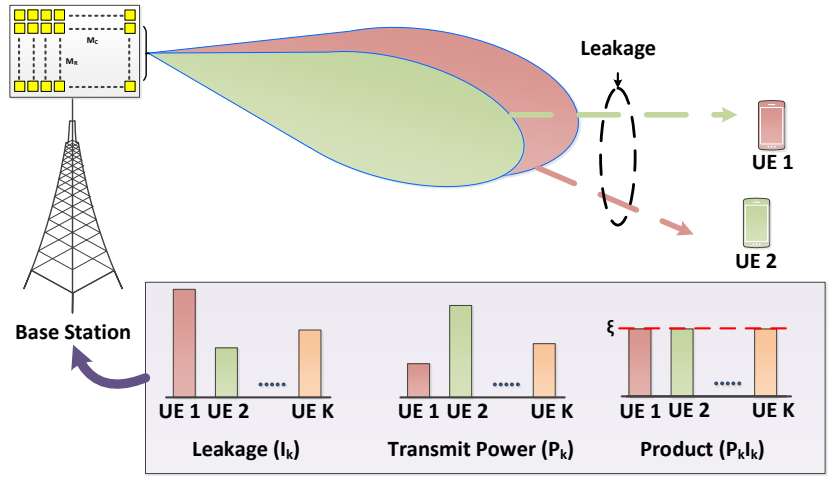

Figure 1. Illustrating the mechanism for the Leakage Based Power Allocation.

the number of channel coefficients to be estimated increases linearly with the number of transmit antennas.

Henceforth, the solution described in [10], which is based on complete knowledge of the propagation channel response, is not suitable for mmWave Massive MIMO systems.

Since the leakage is an evaluation of the power emitted in the direction of the other UEs, so it is possible to avoid the averaging on all the subcarriers by exploiting the angular model of the channel in Equation (7):

$$
I_{k}=P_{k} \sum_{j \neq k}^{K} \sum_{l=1}^{L_{j}} \beta_{j, l}\left|\mathbf{a}_{j, l}^{T} \mathbf{w}_{k}\right|^{2}
$$

Note that if we consider the pure LOS scenario, the dependence on the subcarrier index disappears. Then $N_{F F T}=N_{B c}$ and thus only $M \times K$ coefficients are needed per estimation. This is due to the fact that the 1-Ray channel response is frequency flat over the BW.

Hence, an angle based precoder only needs $2 K$ coefficients (as many azimuth and elevation angles as there are UEs) and also has optimal performance in such an ideal configuration.

\section{HiERARChICAL DBS - LB-PA FrAMEWORK}

\section{A. Overview}

A situation of equity between UEs in terms of level of mutually produced Leakage is achieved by guaranteeing the following equality:

$$
P_{k} I_{k}=\xi \quad \forall k \in[1, K]
$$

where $\xi$ is a constant as shown in Figure 1. This equality is easily achieved by applying the following power per UE:

$$
P_{k}=\frac{\xi}{\sum_{j \neq k}^{K} \sum_{l=1}^{L_{j}} \beta_{j, l}\left|\mathbf{a}_{j, l}^{T} \mathbf{w}_{k}\right|^{2}}
$$

Remark: The value of $\xi$ is chosen so as to ensure the total power constraint $P_{T}$, as follows:

$$
\begin{array}{r}
\sum_{k=1}^{K} P_{k}=P_{T} \longrightarrow \xi=\frac{P_{T}}{\sum_{k=1}^{K} \Lambda} \\
\text {, with } \Lambda=\frac{1}{\sum_{j \neq k}^{K} \sum_{l=1}^{L_{j}} \beta_{j, l}\left|\mathbf{a}_{j, l}^{T} \mathbf{w}_{k}\right|^{2}}
\end{array}
$$




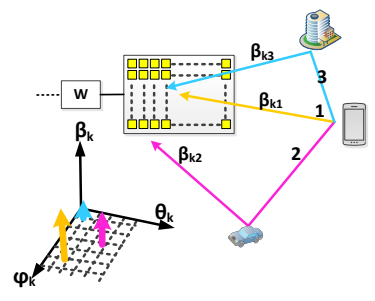

1) DoA + path loss of the $\mathcal{L}_{j}$ paths

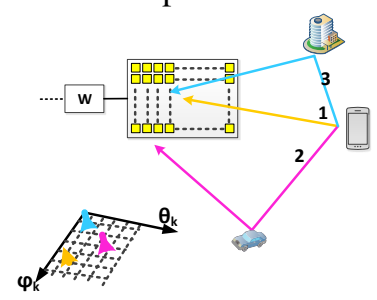

2) DoA of the $\mathcal{L}_{j}$ paths

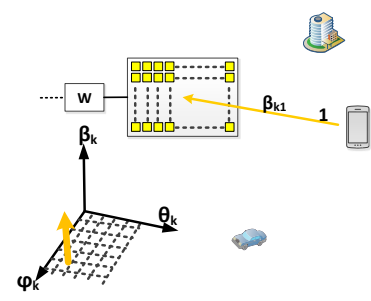

3) DoA + path loss of the LOS

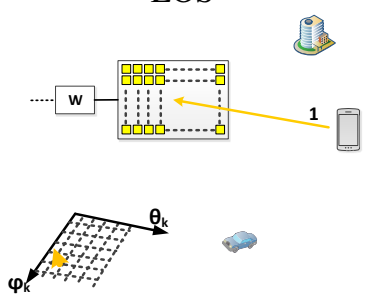

4) DoA of the LOS
Figure 2. Illustration of the amount of information available at the BS, concerning one UE from the $K$ terminals of the cell, for the calculation of the power to be allocated.

\section{B. Simplifying the allocation}

In this section we will describe several simplified expressions of the allocated power $P_{k}$ as a function of the amount of angular information available at the BS. The simulations performed, presented in section VI, show that the performance degradation remains minimal in practical situations. Nevertheless, the underlying overhead reduction is worth interest.

In all the simplified expressions which follow, we will consider the DBS precoder due to its low overhead which is suitable for the future dense networks of small cells. Thus, $P_{k}$ can be expressed as follows:

$$
P_{k}=\frac{1}{\sum_{j \neq k}^{K} \sum_{l=1}^{L_{j}} \beta_{j, l}\left|\mathbf{a}_{j, l}^{T} \mathbf{a}_{k, l}^{*}\right|^{2}}
$$

1) Simplification 1:DoA + Attenuation of direct and secondary paths: Figure 2 - 1) Illustrates the amount of information known to the BS as the number of DoAs and path attenuations for each UE. Each UE $j$ returns back to the BS the information about $T_{j} \leq L_{j}$ paths detected during the estimation process. The expression of the power allocated to the UE $k$ is then:

$$
P_{k}=\frac{1}{\sum_{j \neq k}^{K} \sum_{l=1}^{T_{j}} \beta_{j, l}\left|\mathbf{a}_{j, l}^{T} \mathbf{a}_{k, l}^{*}\right|^{2}}
$$

2) Simplification 2: DoA of direct and secondary paths: Now, if only the DoAs of $T_{j} \leq L_{j}$ paths are known to the BS for each UE $j \in[1, K]$, see Figure 2-2), the power to be allocated to UE $k$ is expressed as:

$$
P_{k}=\frac{1}{\sum_{j \neq k}^{K} \sum_{l=1}^{T_{j}}\left|\mathbf{a}_{j, l}^{T} \mathbf{a}_{k, l}^{*}\right|^{2}}
$$

3) Simplification $3: D o A+$ Direct path attenuation only: This simplification considers only the direct path for each UE, estimating its DoA and its attenuation, as illustrated in Figure 2-3). The power to be allocated to the UE $k$ then becomes:

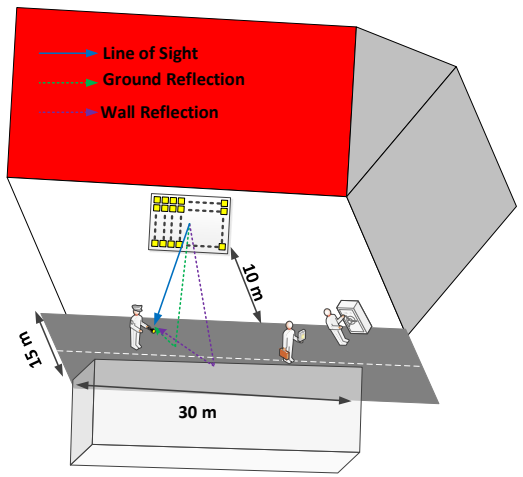

Figure 3. Street canyon environment with illustrating both the 1-Ray Scenario (LOS only) and the 3-Rays scenario (LOS and 2 perfect reflections from the ground and the opposite wall)

$$
P_{k}=\frac{1}{\sum_{j \neq k}^{K} \beta_{j, 1}\left|\mathbf{a}_{j, 1}^{T} \mathbf{a}_{k, 1}^{*}\right|^{2}}
$$

4) Simplification 4: DoA for direct path only: The expression of the allocated power only requires an estimate of the DoA from the BS as shown in Figure 2 - 4), and thus $P_{K}$ is calculated as follows:

$$
P_{k}=\frac{1}{\sum_{j \neq k}^{K}\left|\mathbf{a}_{j, 1}^{T} \mathbf{a}_{k, 1}^{*}\right|^{2}}
$$

\section{Simulation Results}

\section{A. System parameters}

In order to realistically evaluate the performance of the proposed algorithms we develop two specific configurations in a street canyon environment, described in more details in [11]. The first one, called 1-Ray scenario, which only takes into account the LOS path between each UE and the BS. The second one, called 3-Rays, adds two perfect reflections as shown in Figure 3. The simulation parameters are summarized in Table II.

\section{B. Representing simplified power allocation techniques}

The notations used in the legend of Figures $4 \& 5$, displaying the simulation results are as follows:

- Uniform: refers to the Uniform Power Allocation (U-PA).

- Perfect $I_{k}$ : corresponds to the LB-PA allocation with the Leakage known perfectly (i.e. the case in Figure 2 - 1) ).

- DBS DoA and pathloss: corresponds to the DBS precoder with LB-PA allocation given that the Leakage expression is simplified based on the LoS DoA and path loss information only as shown in Figure 2 - 3).

- DBS DoA only: corresponds to the DBS precoder with LB-PA allocation given that the Leakage expression is simplified based on the LoS DoA information only as shown in Figure 2 - 4).

\section{Precoders}

The power allocation coefficients obtained in Equation (17) and its simplifications are applied at the precoding stage, pondering each UE coefficient by its leakage energy as follows: 
Table II

SimUlation PARAMETERS

\begin{tabular}{|c|c|}
\hline Parameter & Value \\
\hline Channel Model & Ray Tracing based [11] \\
\hline Propagation, Scenario & 1-Ray \& 3-Rays, Street Canyon \\
\hline Simulation Type & Monte Carlo (500 realizations) \\
\hline Center Frequency, BW & $60 \mathrm{GHz}, 100 \mathrm{MHz}$ \\
\hline Transmit Antennas, Architecture & 16, Square \\
\hline Antennas Inter-element spacing & $\lambda / 2$ \\
\hline Transmitter height, Receiver height & $10 \mathrm{~m}, 1.5 \mathrm{~m}$ \\
\hline Receive Antennas, UEs' positions & 1, Random \\
\hline
\end{tabular}

$$
\mathbf{W}=\sqrt{P_{T}}\left[\frac{\mathbf{w}_{1}}{\sqrt{I_{1}}}, \frac{\mathbf{w}_{2}}{\sqrt{I_{2}}}, \ldots, \frac{\mathbf{w}_{K}}{\sqrt{I_{K}}}\right] .
$$

To ensure the global transmitted power $P_{T}$ remains unchanged, a normalization factor $\eta$ needs to be applied to form a normalized precoding vector $\mathbf{W}_{n}=\sqrt{\eta} \mathbf{W}$. This factor is expressed as follows:

$$
\eta=\frac{1}{\mathbb{E}\left[\operatorname{Tr}\left(\mathbf{W}^{H} \mathbf{W}\right)\right]} .
$$

We analyze and compare the performance of the 3 aforementioned linear precoders, summed up in Table I, and described in more details in [12]. $\mathbf{H}$ is the normalized channel matrix and does not include the path loss effects as shown in Equation (6). The use of this particular matrix $\mathbf{H}$ instead of $\mathbf{G}$ is to be consistent in our performance comparison, since fairness in relation to UEs' position influenced our choice of the $I_{k}$ expression.

Figure 4 shows how the LB-PA improves the sum SE obtained by the precoders that doesn't consider minimizing the interference (CB and DBS) in the 3-Rays environment. The LB-PA is not applied to the ZF SE analysis, since the role of LB-PA in single cell scenario is to cancel the Inter User Interference (IUI), which is already canceled by ZF in this case leading to $I_{k}=0, \forall k \in[1, K]$. The results in Figure 4 show that low complexity precoders (CB and DBS) can achieve almost the same sum SE as ZF, and they can even achieve higher $\mathrm{SE}$ when the number of UEs increases in dense environments. This result shows that with the low complexity-low overhead framework based on the DBS, together with LB-PA we can achieve better SE performance compared to ZF with less complexity, less overhead, and without matrix inversion limitations. Table III shows that our proposed DBS - LB-PA solution has much less complexity and less overhead compared to ZF. Moreover, it requires only $2 K$ channel coefficients against $\frac{M \times K \times N_{F F T}}{N_{B c}}$ in case of CB - LB-PA solution.

Impact of $L B-P A$ on the system performance: The $\mathrm{CB}$ benefits from the diversity of the channel as it forms a beam adapted to it, while the DBS knows only the direction of the UEs and not their channels. However, according to Figure 4 with LB-PA the gap between DBS and CB decreases. Moreover, LB-PA succeeds in decreasing the gap significantly between $\mathrm{CB}$ and $\mathrm{ZF}$. These results unleash the main advantage of LB-PA, which is reducing the benefit of using high complexity-high overhead signal processing techniques.

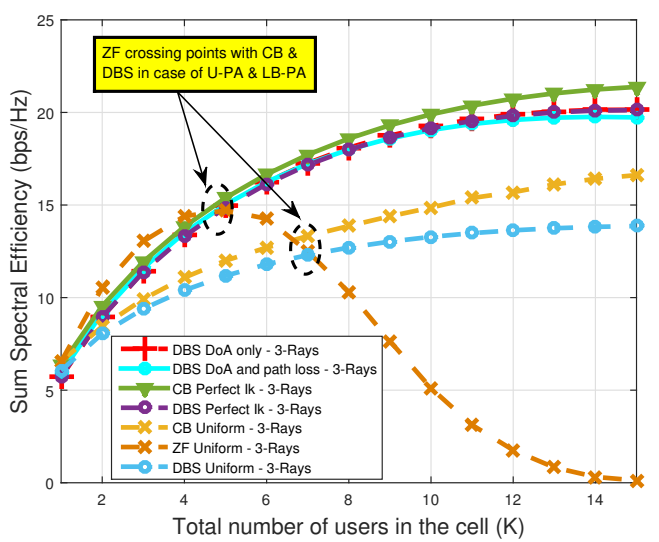

Figure 4. Sum SE of CB, ZF and DBS precoders assuming perfect CSIT or DoA knowledge in the 3-Rays environment. The SE is averaged over 500 random UEs' positions for each $K \in[1,15]$ with $M=16$.

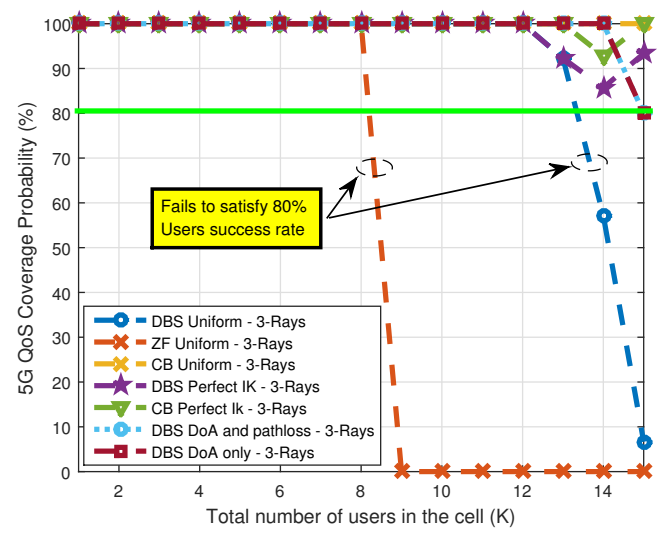

Figure 5. Coverage probability of $\mathrm{CB}, \mathrm{ZF}$ and DBS precoders assuming perfect CSIT or DoA knowledge in the 3-Rays environment. The coverage probability is averaged over 500 random UEs' positions for each $k \in[1,15]$ with $M=16$.

For the DBS precoder, simplifications 1,3 and 4 respectively as described in Section $\mathrm{V}$, are intertwined with the curve which shows the CB performance with LB-PA based on perfect CSIT. Therefore, we can conclude that, by knowing the directions of the direct paths for each UE, we can achieve high SE with low overhead.

Comparing the results to the ZF baseline precoder we can see that the crossing point (number of UEs above which the $\mathrm{CB}$ and DBS outperforms the ZF) is quite higher with U-PA than with LB-PA ( $\sim 7$ UEs with U-PA against $\sim 5$ UEs with LB-PA).

In order to evaluate the coverage probability and the Quality of Service (QoS) satisfaction for all the UEs in the cell, we first define a QoS constraint on per UE data rate. According to [14] the average UE data rate in $5 \mathrm{G}$ should be at least $0.1 \mathrm{Gbps}$. In our simulations we define the acceptable outage probability to be $P_{\text {out }}=20 \%$. Thus the cell coverage probability should be at least $80 \%$. Figure 5 gives more insights in terms of coverage and cell edge UEs' performance. We can observe that for all the precoders based on the LB-PA the 5G per UE QoS constraint is satisfied for at least $80 \%$. However, for the ZF, DBS precoders with U-PA the 5G per UE QoS constraint isn't satisfied with increasing the number 
Table III

COMPUTATIONAL COMPLEXITY AND OVERHEAD

\begin{tabular}{|c|c|c|c|c|}
\hline Algorithm & ZF [13] & CB [13] & DBS & LB-PA [10] \\
\hline \multirow{2}{*}{ Complexity } & $K\left(24(K-1) M^{2}\right.$ & & & $K(12 M+4)$ \\
& $+48(K-1)^{2} M+54(K-1)^{3}+6 M$ & $K(6 M+2(M-1)+6 M)$ & $K(6 M+2(M-1)+6 M)$ & \\
\hline Coefficients & $\frac{M K N_{F F T}}{N_{B C}}$ & $\frac{M K N_{F F T}}{N_{B C}}$ & LOS DoA based= $2 K$ & LOS DoA based= $2 K$ \\
\hline
\end{tabular}

of simultaneously served UEs per cell ( $~ 9$ UEs in the case of ZF and $\sim 14$ in the case of DBS). Henceforth, the results reinforce our assumption that LB-PA applies some sort of fairness between the served UEs. This is due to forcing the total leakage emitted by each UE to be constant as shown in Equation (14) and Figure 1. Thus, LB-PA increases the system sum SE in a non greedy behavior, by considering the constant leakage per UE linear constraint.

\section{CONCLUSION}

In this paper, we have demonstrated how the DoA information makes an assessment of the interference in a MU transmission. We have derived simple interference-wise power allocation solutions based on the angular channel structure to relax the overhead requirements. Henceforth, the advantages of our DBS - LB-PA framework can be summarized as follows:

- It is more suitable for MU MIMO LoS dominated systems with partial channel knowledge compared to traditional complex solutions (such as ZF).

- It ensures a relative threshold of fairness between the UEs, due to minimizing the leakage.

\section{ACKNOWLEDGMENTS}

This work has received a French state support granted to the CominLabs excellence laboratory and managed by the National Research Agency in the Investing for the Future program under reference Nb. ANR-10-LABX-07-01 and project name $\mathrm{M}^{5} \mathrm{HESTIA}$ (mmWave Multi-user Massive MIMO Hybrid Equipments for Sounding, Transmissions and HW ImplementAtion). Part of the ideas have been investigated in the framework of research collaborations with the Institute of Research and Technology IRT $b<>$ com.

\section{REFERENCES}

[1] J. F. Monserrat, H. Droste, O. Bulakci, J. Eichinger, O. Queseth, M. Stamatelatos, H. Tullberg, V. Venkatkumar, G. Zimmermann, U. Dötsch, and A. Osseiran, "Rethinking the mobile and wireless network architecture: The metis research into 5g," in 2014 European Conference on Networks and Communications (EuCNC), June 2014, pp. 1-5.

[2] E. G. Larsson, O. Edfors, F. Tufvesson, and T. L. Marzetta, "Massive mimo for next generation wireless systems," IEEE Communications Magazine, vol. 52 no. 2, pp. 186-195, February 2014.

[3] M. Xiao, S. Mumtaz, Y. Huang, L. Dai, Y. Li, M. Matthaiou, G. K. Karagiannidis, E. Björnson, K. Yang, C. Lin, and A. Ghosh, "Millimeter wave communications for future mobile networks," CoRR, vol. abs/1705.06072, 2017.

[4] S. Rangan, T. S. Rappaport, and E. Erkip, "Millimeter-wave cellular wireless networks: Potentials and challenges," Proceedings of the IEEE, vol. 102, no. 3 , pp. 366-385, March 2014.

[5] W. Fang, H. Sun, and L. Yang, "Power allocation for maximizing sum capacity of multiuser mimo downlink with transmit precoding based on slnr," in 2011 IEEE 73rd Vehicular Technology Conference (VTC Spring), May 2011, pp. 1-5.

[6] J. Jang, K. B. Lee, and Y.-H. Lee, "Transmit power and bit allocations for ofdm systems in a fading channel," in Global Telecommunications Conference, 2003. GLOBECOM '03. IEEE, vol. 2, Dec 2003, pp. 858-862 Vol.2.

[7] J. Zhang, Y. Jiang, P. Li, F. Zheng, and X. You, "Energy efficient power allocation in massive mimo systems based on standard interference function," in 2016 IEEE 83rd Vehicular Technology Conference (VTC Spring), May 2016, pp. 1-6.

[8] L. Zhao, H. Zhao, F. Hu, K. Zheng, and J. Zhang, "Energy Efficient Power Allocation Algorithm for Downlink Massive MIMO with MRT Precoding," in Vehicular Technology Conference (VTC Fall), 2013 IEEE 78th, Sep. 2013, pp. 1-5.

[9] I. K. Choi and S. K. Lee, Method and apparatus for allocating transmission power in multi input multi output system. Google Patents, Nov. 2014, uS Patent 8,891,643.

[10] L. Dong, G. Wu, H. Feng, and S. Li, "Leakage-based power allocation for relaxed SLNR beamforming in multi-cell MU-MIMO systems," in 2014 IEEE International Conference on Communication Systems (ICCS), Nov. 2014, pp. $512-516$.

[11] A. Roze, M. Helard, M. Crussiere, and C. Langlais, "Linear Precoder Performance for Massive MIMO Systems in near LOS Environments: Application to mmWave Transmission," in European Wireless 2015; 21th European Wireless Conference; Proceedings of. VDE, 2015.

[12] A. Rozé, M. Helard, M. Crussiere, and C. Langlais, "Millimeter-Wave Digital Beamsteering in Highly Line-of-Sight Environments for Massive MIMO Systems," in Wireless World Research Forum Meeting 35, 2015.

[13] C. S. Park, Y. S. Byun, A. M. Bokiye, and Y. H. Lee, "Complexity reduced zero-forcing beamforming in massive mimo systems," in 2014 Information Theory and Applications Workshop (ITA), Feb 2014, pp. 1-5.

[14] "IMT vision - framework and overall objectives of the future development of IMT for 2020 and beyond," M.2083-0, International Telecommunication Union - ITU, Sep. 2015. 